\title{
The on-line detection of moisture and moist coatings by means of thermal waves
}

\author{
by R. Hüttner and E. Schollmeyer \\ Deutsches Textifforschungszentrum Nord-West e.V., Institut an der Universität Duisburg \\ Gesamthochschule, Frankenring 2, D-47798 Krefeld
}

\begin{abstract}
The method of thermal wave detection by infrared radiometry is applied to characterize physical properties of moist fabrics. Especially the determination of moisture content on moving fabrics and the control of layer thicknesses in continuous industrial production processes are investigated systematically. The results show a good correlation between the photothermal signal and the moisture content. The thickness of a thin coating could be determined by frequency scans and the thickness of moist coatings could be controlled in a continuous process.
\end{abstract}

\section{Introduction}

As photothermal methods have the potential to do non-contact non-destructive measurements, they were applied to a wide range of problems of measuring and controlling in industry $[1,2]$. Because of their depth sensitive detection especially the measurement of layers is a frequent task, which is an important application in the industry as well. Common techniques, like for example the IR-Reflectance technique, mainly only give information about surface properties and cannot measure physical properties in the depth. For this purpose, as for example for measuring the thickness of a layer, photothermal methods are ideal candidates.

In a common photothermal setup the measurements are done with fixed samples in a static arrangement, whereas a lot of interesting industrial problems deal with moving matter in continued processes.

Busse and Eyerer first mentioned measurements of thermal waves with rotating disks in a laboratory setup [3]. We have already reported that photothermal measurements may be used to determine the moisture content of textiles even at moving samples in industrial processes and to characterize effects of thermal treatments onto thermal properties $[4,5]$. Here we show new results obtained from painted samples and from measurements, where the thickness of a moist coating could be controfled even in running processes.

\section{Experimental}

A $100 \mathrm{~W}$ mercury-vapour lamp was used to generate thermal waves, which allows a low cost experimental setup and furthermore an application of a large heating spot of about 10 $\mathrm{mm}$ of diameter. This prevents an overheating of the sample by lowering the radiation intensity. Furthermore this eliminates 3-dimensional effects as the detection spot of the infrared optics is much smaller ( $3 \mathrm{~mm}$ of dia.). For the detection of the thermal waves a $\mathrm{HgCdTe}$ detector has been used, whose signals were amplified with the help of a lock-in amplifier. See $[8,9]$ for a more detailed description. A schematic of the experimental setup is shown in figure 1 . The part on the grey background is the experimental device, which is transportable and can be built into an industrial machine. 


\section{Measurements and results}

\subsection{Measurements of the Moisture content}

Figure 2 shows photothermal amplitudes obtained from static samples during a drying process and mirrors the influence of moisture onto the thermal properties of the sample. The dots $\left({ }^{*}\right)$ represent the moisture content and $(X)$ the photothermal amplitude obtained from a polyester fabric. The amplitude increases with decreasing moisture content until it becomes constant, when the sample becomes dry (here: after $55 \mathrm{~min}$ ). This can be explained by the fact that the thermal conductivity decreases (and so does the thermal effusivity e) when the moisture content decreases. This causes an increasing of the photothermal amplitude, which is given by l/e in a first approximation [8]. This is confirmed with calculations for the thermal effusivity e with a model basic on effective medium theory [6], where a mixing of the thermal properties of water and polyester is considered [9]. Figure 3 shows the same effect, now obtained from on line measurements with moving fabrics, where the moisture content was varied in steps. The influence of moisture and so generally of the thermal properties on the signal obtained from moving fabrics may be different, because the movement causes a modification of the signal generation process. In fact, in order to obtain an exact theoretical description for the photothermal signal from moving samples a more complex signal theory has to be used, as demonstrated by Netzelmann [7]. Then the absolute photothermal amplitude depends on the sample velocity. When the velocity is kept constant, the dependence from the moisture content remains. In order to compare the signal dependencies from the moisture content for each kind of measurement the photothermal amplitudes are plotted directly versus the moisture content (figure. 4). These curves, which serve as calibration curves and can be used to determine the moisture content directly from the measured signal [4], look quite similar and show the same characteristics. This shows that the movement does not disturb the general dependence between the photothermal signal and the thermal properties, which therefore can well be determined in continuous industrial processes.

\subsection{Characterisation of layers and thickness control}

Frequency dependent photothermal measurements obtained from polyurethane coatings on a white paper board are shown in figure 5 . The thickness of the layers was $61 \mu \mathrm{m}$ and $24 \mu \mathrm{m}$ respectively. The amplitudes were normalized with values obtained from a black board with a thickness of $0.55 \mathrm{~mm}$. Theoretical curves, which were calculated with a model for samples consisting of two layers of finite thickness with inner heat sources (non infinite absorption coefficient), are plotted in solid lines. The curves show that the chosen model mirrors the experimentally observed behaviour with a suitable choice of parameters. It should be noted that this observation could not necessarily be expected, because the thermal properties of board are mostly unknown and the surface roughness of the board produces huge changes in the thermal properties of the polymer board interface. This effect has to be taken into account when fitting theoretical curves for the photothermal phases (not shown here). With well known thermal parameters, which have been determined once, the measurements allow the determination of the polymer layer thickness.

For controlling industrial processes in many cases the detection of changes in process parameters is sufficient and the absolute value (for example of the layer thickness) is not important, especially if the value has been controlled at the beginning of the process. Figure 6 shows an example of such a case, where the aim was to control the thickness of a moist coating on a textile substrate in a real industrial application. For this purpose the photothermal setup was built into a laminating machine, where the thickness of the coating changed with time due to changes in instable machine parameters. The photothermal signals are plotted as a function of time and show a clear dependence of the coating thickness. 


\section{Conclusions}

The detection of thermal waves by photothermal radiometry has been shown to be a powerful tool for the controlling the moisture in moving fabrics in continuos industrial processes and the thickness of coatings. Generally this method may serve to characterize thermal properties of polymer coatings and to control machine parameters in continuous production processes. It seems to be promising to look for further applications in this field.

\section{REFERENCES}

[1] See different articles and authors in the conference proceedings Photoacoustic and Photothermal Phenomena III - Conference, Ed. D. Bicanic, Springer-Verlag Berlin, Heidelberg, 1992.

[2] Eurotherm Seminar on Quantitative Infrared Thermography (QIRT), 19-22. Aug.1994, Sorrento, Italy, Edition Européennes Thermique et Industrie, Paris, 1995.

[3] BUSSE (G.) und EYERER (P.) - Thermal wave remote and nondestructive inspection of polymers. Appl. Phys. Lett. 43, 1983, p. 355-357,.

[4] HÜTTNER (R.) SCHOLLMEYER (E.) - Photothermal infrared radiometry applied to textile materials - General characterization and determination of moisture content. Eurotherm Seminar on Quantitative Infrared Thermography (QIRT), 19-22 .Aug.1994, Sorrento, Italy, Edition Européennes Thermique et Industrie, Paris, 1995, pp. 226-229.

[5] HÜTTNER (R.), BAHNERS (T.), SCHOLLMEYER (E.), WEBER (A.), BECKER (W.), BEIN (B.K.), PELZL (J.) - Modulated photothermal infrared radiometry as a tool for on-line characterization of polymeric coatings in textile processes. Proc. of the $9^{\text {th }}$ Int. Conf. on Photoacoustic and Photothermal Phenomena, June 27-30, Nanjing, P.R. China, Vol. 6 Supplement, 1996, pp. 735-738.

[6] BRAILSFORD (A. D.), MAJOR (K.G.) - Thermal conductivity of aggregates of several phases, including porous material. Brit. Appl. Phys., Vol.15, 1964, p. 313-319

[7] NETZELMANN (U.). - Analysis of thermal wave propagation in coatings on moving objects. Proc. of the $9^{\text {it }}$ int. Conf. on Photoacoustic and Photothermal Phenomena, June 27-30, Nanjing, P.R. China, Vol. 6 Supplement, 1996, pp. 235-238

[8] HÜTTNER (R.), BOSSMANN (A.), SCHOLLMEYER (E.) - Photothermische Radiometrie zur Messung der Warenfeuchte an laufenden Materialien. Textilveredelung, Schweiz, 29. Okt. 1994, p.295-300

[9] HÜTTNER (R.) - Dissertation, Ruhr-University of Bochum, ISBN 3-931 333-02-7, 1996 


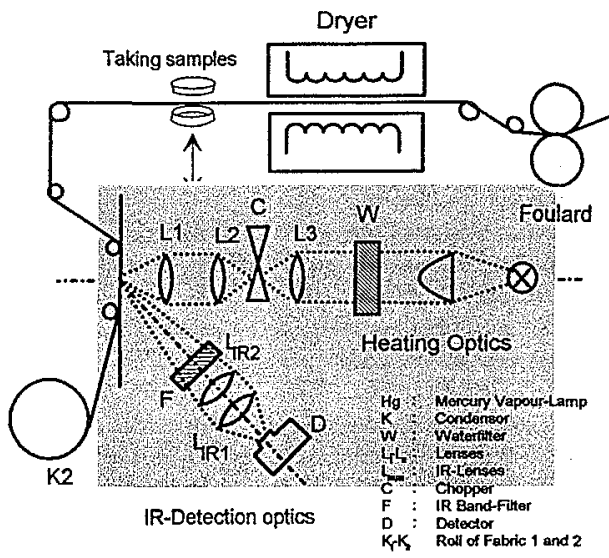

Fig. 1: Experimental setup for the detection of thermal waves in a continuous process.
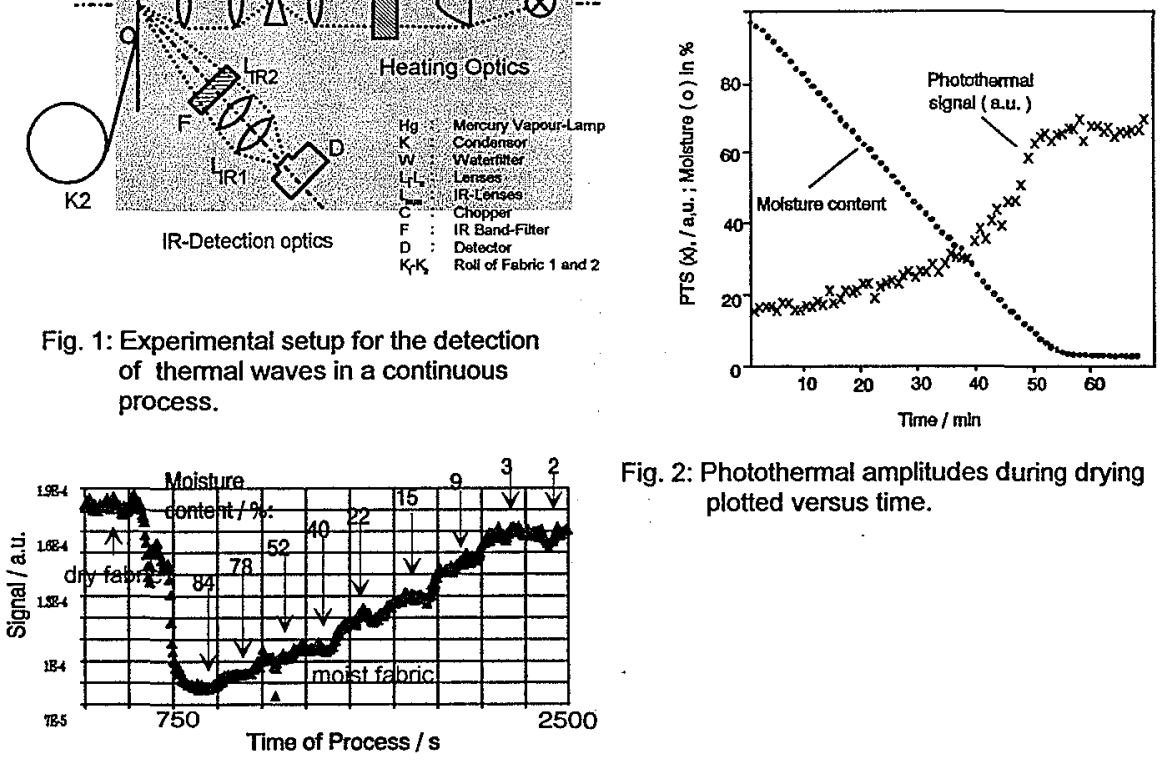

Fig. 2: Photothermal amplitudes during drying plotted versus time.

Fig. 3: Photothermal amplitudes obtained from a brown dyed PETP sample at different states of drying.

moving samples
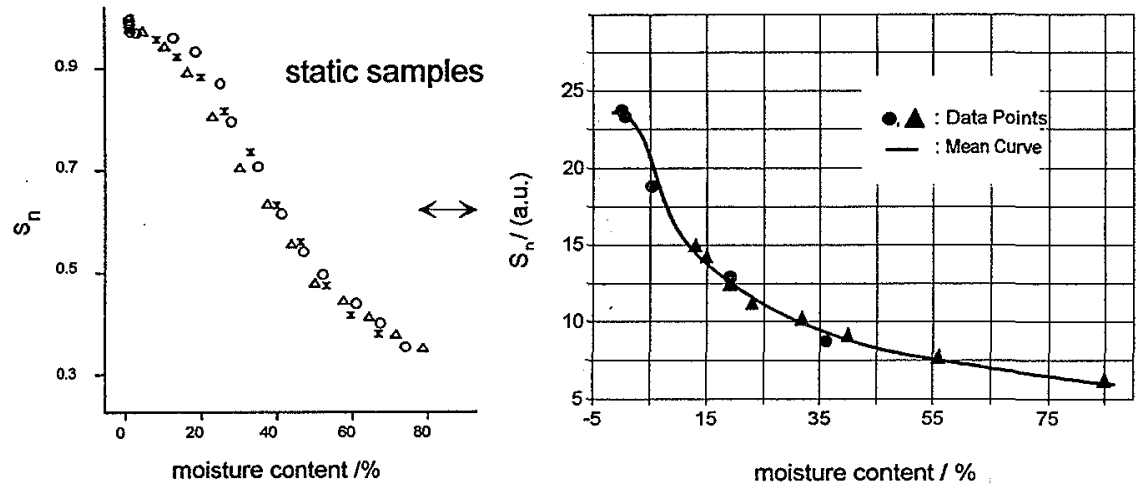

Fig.4: A comparison between the photothermal signals obtained from static and from moving samples. 


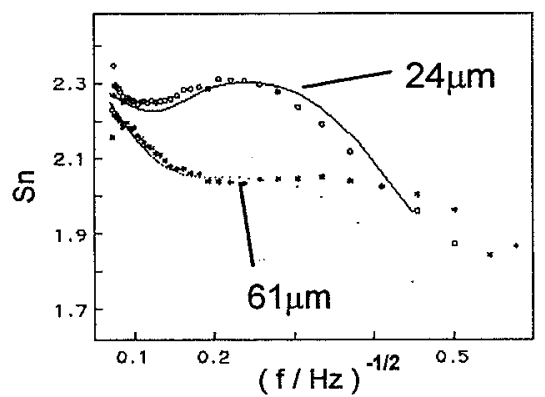

Fig. 5: Frequency dependent normalized photothermal amplitudes obtained from different thickness polyurethane coatings and calculated curves.

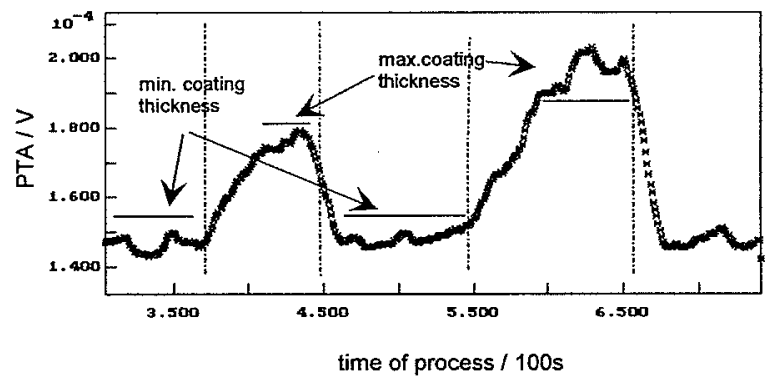

Fig. 6: Photothermal amplitudes obtained from moving fabrics with different thickness dispersion coatings. 